\title{
Integrating S.PSS and DE
}

\author{
Ranjani Balasubramanian, Carlo Vezzoli, Fabrizio Ceschin, Jacob Matthew, \\ Abhijit Sinha, and Christoph Neusiedl
}

\section{Introduction to S.PSS Applied to DE: Sustainable Opportunities}

The combination of Sustainable Product-Service Systems (S.PSS) and Distributed Economies (DE) has been considered as a promising mode of developing sustainability through regional resilience and by empowering a shift to a more localized economic model [11]. Especially in regions with significant middle- and low-income populations, DE provides possibilities for increased localized employment generation, and many such schemes have been implemented in both urban and rural areas. In underserved regions, this could help decrease emigration and develop better services in these economies. The LeNSin project studied the shift to S.PSS as a mode of DE designing and delivering. The win-win sustainability benefits could be summarized as follows.

S.PSS is a promising approach to diffuse DE in low/middle-income (all) contexts, because it reduces/cuts both the initial (capital) cost of DE product/equipment purchasing (that may be unaffordable) and the running cost for maintenance, repair, upgrade, etc. of such DE hardware (that may cause

R. Balasubramanian ( $₫)$

Srishti Institute of Art, Design and Technology, Bengaluru, India

e-mail: ranjani.b@srishti.ac.in; ranjani.bms@gmail.com

C. Vezzoli

Design Department, Politecnico di Milano, Milan, Italy

F. Ceschin

Department of Design, Brunel University London, London, UK

J. Matthew

Industree Foundation, Bengaluru, India

A. Sinha $\cdot$ C. Neusiedl

Project DEFY, Bengaluru, India

(C) The Author(s) 2021

C. Vezzoli et al. (eds.), Designing Sustainability for All, Lecture Notes

in Mechanical Engineering, https://doi.org/10.1007/978-3-030-66300-1_3 


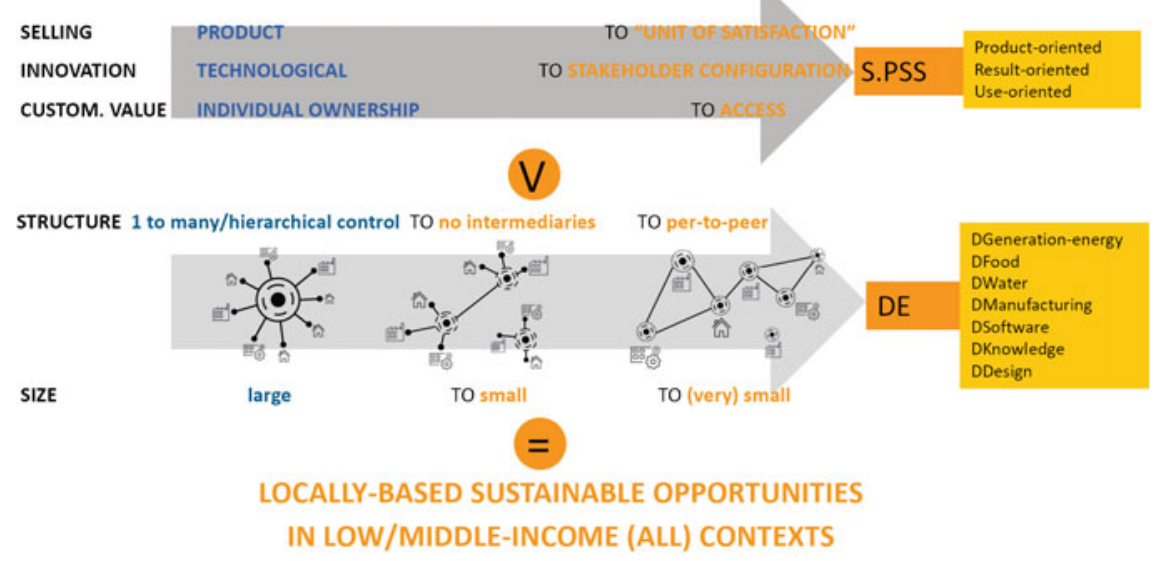

Fig. 1 The coupling of the two win-win sustainability paradigm shifts represented by S.PSS and DE

the interruption of use) increasing local employment and related skills. Furthermore, by offering a DE system adopting an S.PSS model, the producer/provider is economically incentivized to design low environmentally impacting DE products/equipment. Finally, S.PSS applied to DE is a promising key leverage for a sustainable development process for all aiming at democratizing access to resources, goods and services.

These win-win potentials are based, in fact, on the coupling of the two winwin sustainability paradigm shifts of S.PSS and DE we have already discussed in the previous chapters of S.PSS and DE we have already discussed in the previous chapters (see Fig. 1):

1. The shift from a traditional product sale model to S.PSS, i.e. the shift of customer perceived value from individual ownership to access to a mix of products and services (systems) fulfilling a given unit of satisfaction;

2. The shift from centralized to decentralized/distributed systems in which a smallscale unit of production is locally based, i.e. nearby or at the point of use, and where the user can become a producer.

Further consideration could be made in relation to the increased access to the internet and digital infrastuctures and tools combined with the projected development of distributed technologies, such as 3D printing, which significantly increase the potential and ease of setting up these Distributed Economies. In areas with low income, even basic internet penetration has opened up possibilities to access knowledge and know-how to set up distributed networks. A number of organizations and governments are supporting the set-up of such networks in low- and middle-income regions, and the main focus is to develop affordable systems with the aid of technology that requires lower investment cost. In middle- to higher-income regions, 
the likelihood of using more capital-intensive processes (like 3D printing manufacturing) is higher and there is a push to develop DE networks with the aid of technology. However, it seems that technology and access to information sharing systems will be key to developing scalable and replicable DE. With the accelerated pace of technological penetration, it is possible to envision what an S.PSS would look like applied in a DE format.

Sustainable value-adding PSS can only be created taking into account every life cycle stage of products and services [8]. Distributed Manufacturing, for example, applied to near-future scenarios addresses each S.PSS life cycle stage, thus showing the potential to improve PSS development from the sustainability point of view:

- The design stage predominantly benefits from collaboration between PSS provider and customer, enabled by connectivity through digital channels and physical interaction in local production facilities, which results in better S.PSS acceptance.

- The material production (pre-production) and production stages benefit from the distribution of manufacturing facilities, equipped with digitally connected manufacturing technology. The ability to send digital production files to remote locations, for example, allows PSS companies to produce products and spare parts in close proximity to customers and/or resources, thus reducing the environmental impact of distribution.

- The use stage is supported with the largest number of near-future scenarios tackling on-site and on-time provision of maintenance services and empowering customers to maintain, repair, update, upgrade and re-manufacture the products included in the S.PSS solution.

- The end-of-life phase is facilitated by the application of sensor technology, which helps to indicate products' and components' end-of-life by alerting PSS providers and customers. Finally, a distributed network of localized recycling facilities eases product collection, recycling and/or energy recovery.

This chapter examines case studies of S.PSS applied to DE (both Distributed and Decentralized production units) from across the globe. It is important to note nevertheless that it is challenging to clearly define and categorize the case studies, as most of them consist of varying degrees of PSS or DE with different types of interactions. These could, however, be used to develop a categorical understanding of S.PSS applied to DE.

\section{Case Studies of S.PSS and DE Integration}

\subsection{S.PSS and Distributed Energy Generation (DG)}

As discussed in Chap. 2, Distributed energy Generation (DG) represents a promising strategy to provide energy access with a range of sustainability benefits. However promising, the implementation of DG solutions should not only focus on the technical 


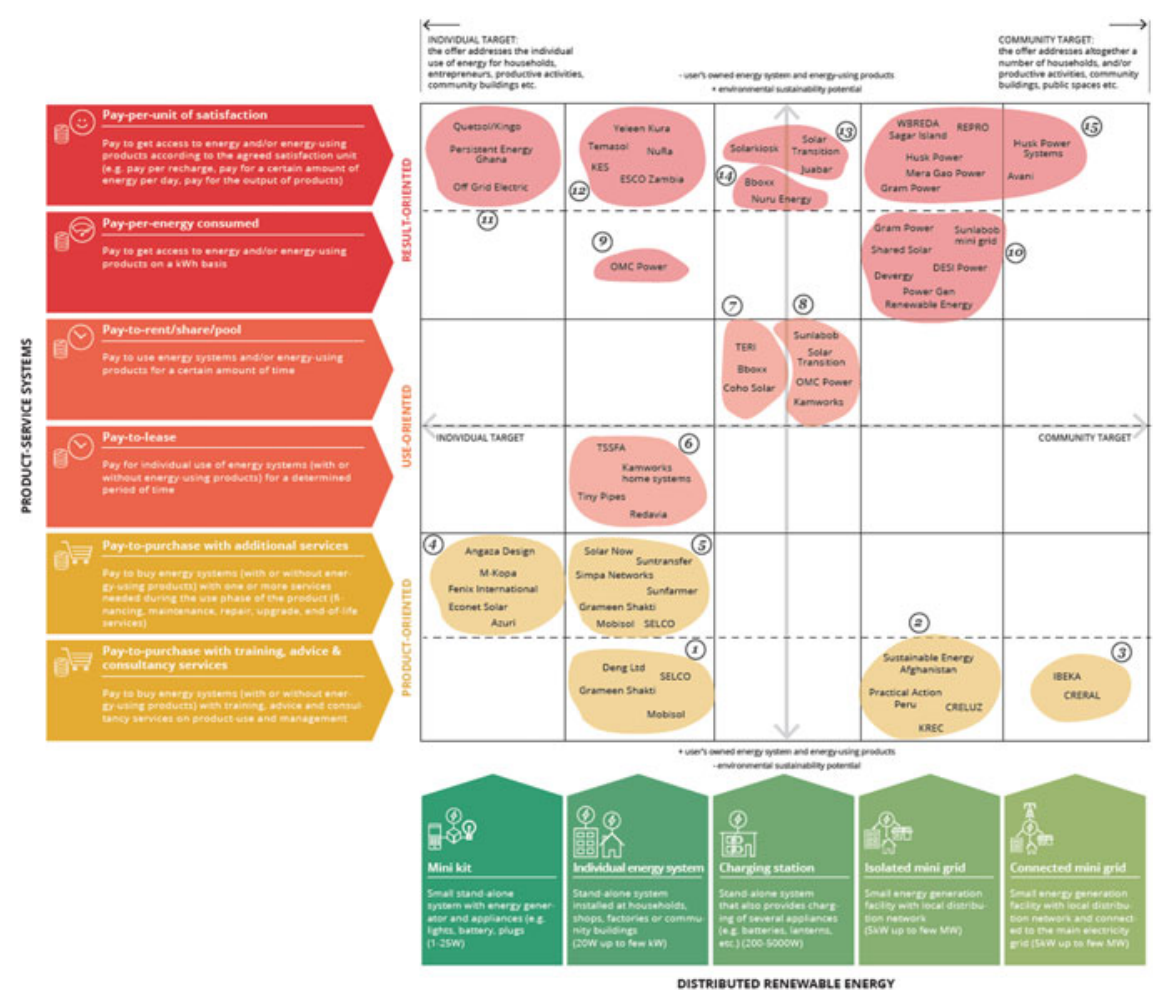

Fig. 2 Archetypal models of PSS applied to DG in low- and middle-income contexts [2, 3]

aspects. There are other aspects which are crucial for the success of DG solutions. Most of the unsuccessful cases of DG are linked to problems such as the lack of a maintenance and repair network, lack of understanding of user needs or lack of a proper business model $[3,10]$. For this reason, an S.PSS system design approach should be adopted. This means that, in addition to energy technology, the stakeholder value chain, the product-service combination and the business model aspects should be taken into consideration and integrated into systemic solutions [6, 9]. S.PSS applied to DG can be categorized in 15 archetypal models (Fig. 2) [2, 3]:

1. Selling individual energy systems with advice and training services;

2. Offering advice and training services for community-owned and-managed isolated mini-grids;

3. Offering advice and training services for community-owned and-managed connected mini-grids;

4. Selling mini-kits with additional services;

5. Selling individual energy systems with additional services;

6. Offering individual energy systems (and energy-consuming products) in leasing;

7. Renting energy-using products through entrepreneur-owned and-managed charging stations; 
8. Renting energy-using products through entrepreneur- or community-managed charging stations;

9. Offering access to energy (and energy-using products) on a pay-perconsumption basis through individual energy systems;

10. Offering access to energy (and energy-using products) on a pay-perconsumption basis through isolated mini-grids;

11. Offering access to energy \& energy-using products on a pay-per-unit of satisfaction basis through mini kits;

12. Offering access to energy (and energy-using products) on a pay-per-unit of satisfaction basis through individual energy systems;

13. Offering access to energy-using products through community- or entrepreneurmanaged charging stations on a pay-per-unit of satisfaction basis;

14. Offering recharging services through entrepreneur-owned \& -managed charging stations;

15. Offering access to energy (and energy-using products) on a pay-per-unit of satisfaction basis through mini-grids.

Several case studies of S.PSS and Distributed energy Generation (DG) are presented below.

\section{SELCO (example of archetypal model 1 and 5)}

Active since: 1995

Provider/s: SELCO and local community agents

Customers: Rural Households/Communities, Institutions

S.PSS Type: Product-oriented S.PSS

DE configuration: Distributed and Decentralized energy Generation

Products: Solar Home Lighting, Solar Water Heater, Solar Inverter Systems,

DC Home Appliances like Butter Churners, Grinders, etc.

Services: Product customization, installation, maintenance and repair, community training, tailoring financing options, advisory and capacity building.

Payments: Pay for product-service-system

Resource: Solar Energy

Location: India

SELCO is a rural energy service social enterprise that provides affordable and environment-friendly energy services to rural households. SELCO produces solar Product-Service Systems for individuals, communities or institutions. The ultimate aim of the company is to provide affordable rural electrification through renewable sources and to achieve this, SELCO provides services that include financing consultancy, customized product configurations, training, maintenance and repair.

The company also creates additional distributed economies by training local youth for maintenance of the systems, supporting local entrepreneurs who can buy the 


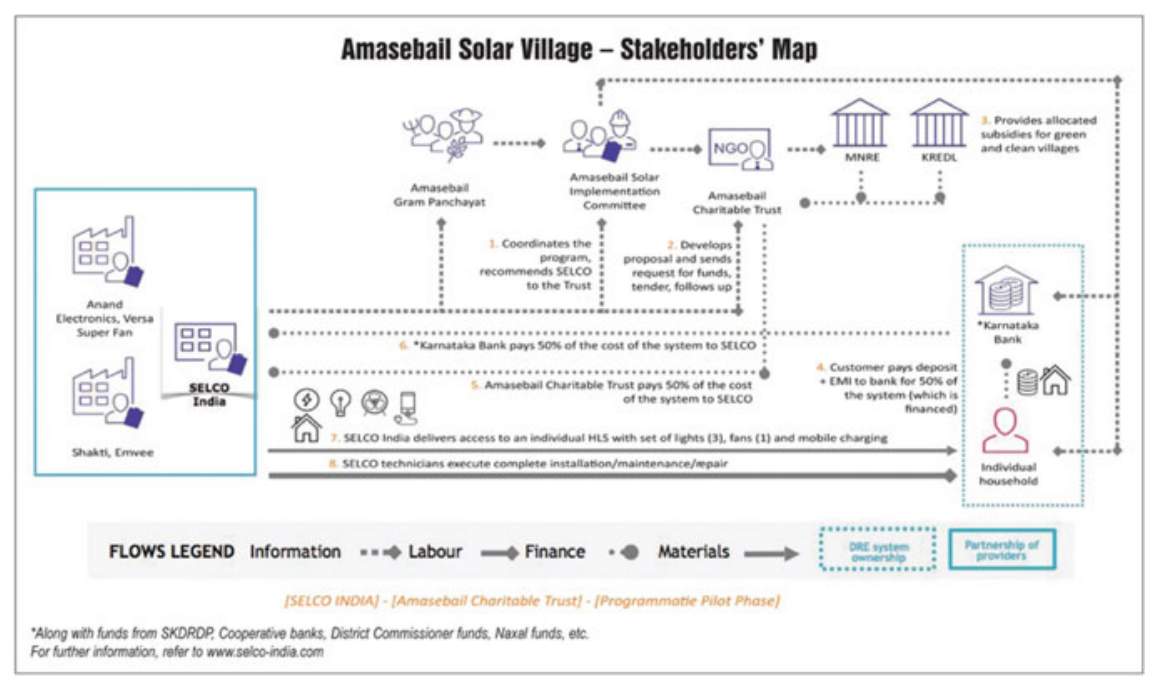

Fig. 3 System map of a SELCO S.PSS for a village in India. (Source: renewablewatch.in)

system and develop a livelihood by providing charging services and connecting them to financial services. The company has also diversified into producing solar energy-powered products like sewing machines and photocopy machines which can further develop into opportunities for distributed manufacturing.

The most striking characteristic of the company is its targeted user group and diversified Product-Service System in a standard distributed format, involving multiple relevant stakeholders (Figs. 3 and 4).

\section{Solarkiosk (example of archetypal model 15)}

Active since: 2011

Provider/s: Solarkiosk Solutions GmbH (E-HUBB and related equipment); local subsidiary (installation, maintenance and repair)

Customers: Solarkiosk local subsidiaries (own model), international organizations (B2B)

S.PSS Type: Use-oriented (B2B), Result-oriented (B2C)

DE configuration: Decentralized energy Generation

Products: E-HUBB, Solar Pico systems, Solar Home Systems, PAYG systems, other products

Services: Project based design, production, installation, maintenance, engineering Payments: E-HUBB is in ownership of Solarkiosk (own model), Project budgets (B2B sales) 


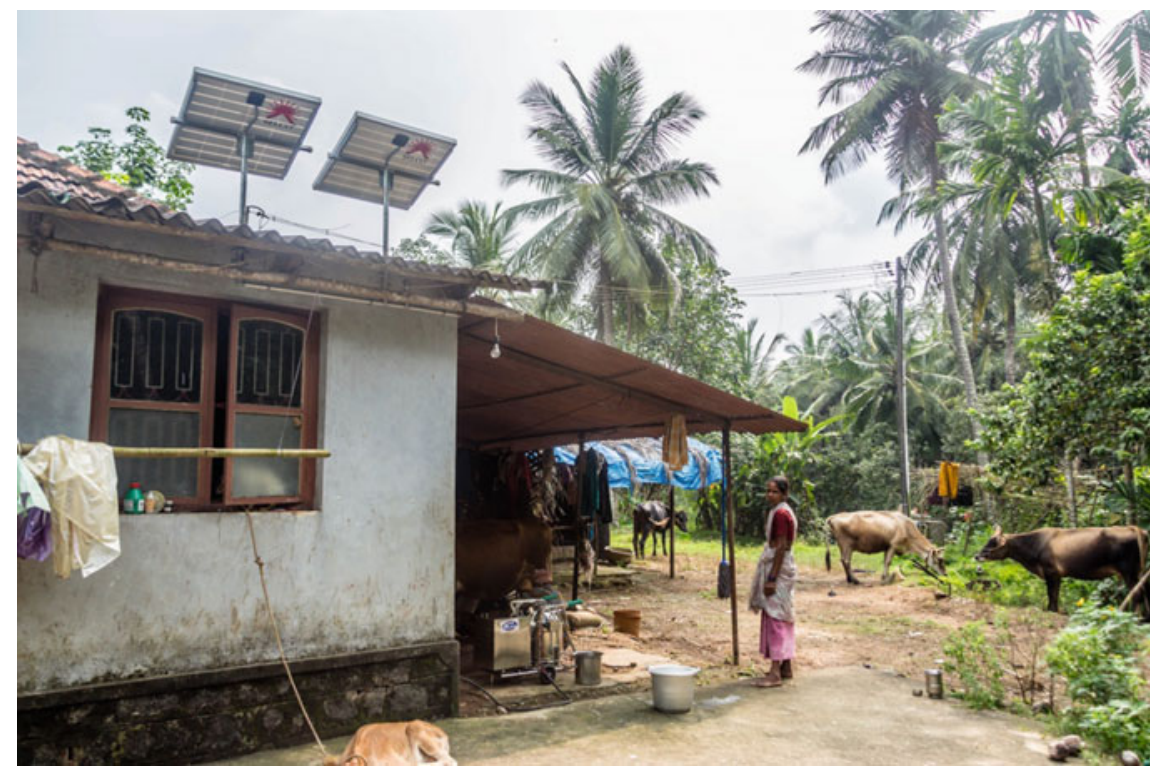

Fig. 4 Solar powered cow milking unit. (Source SELCO)

Payments: E-Hubb is given for free (B2B), Pay per use (B2C)

Resource: Solar Energy

Location: Ethiopia, Kenya, Rwanda, Tanzania, projects realized in 11 other countries

The company targets local entrepreneurs, especially women, for the provision of energy services through charging stations. Solarkiosk designs and installs the EHubb, a charging station provided with solar panels and energy-consuming products and recruits a local entrepreneur who manages the system and appliances. Due to the modular configuration of the station, he/she can provide a wide range of energydependent services such as internet connectivity, water purification, copying, printing and scanning. Customers pay for the service they need: pay to print, pay to get purified water, pay for internet access and other services (Figs. 5 and 6).

\subsection{S.PSS and Distributed Food Production (DF)}

If small-scale Food production units (DF) are connected with other DF (e.g. to share food overproduction), they become a Distributed Food Production Network (DFN), which may in turn be connected with nearby similar networks. If properly designed they hold promise to promote locally based sustainability, i.e. Sustainable Distributed Food production (S.DF) systems. 
SOLARKIOSK

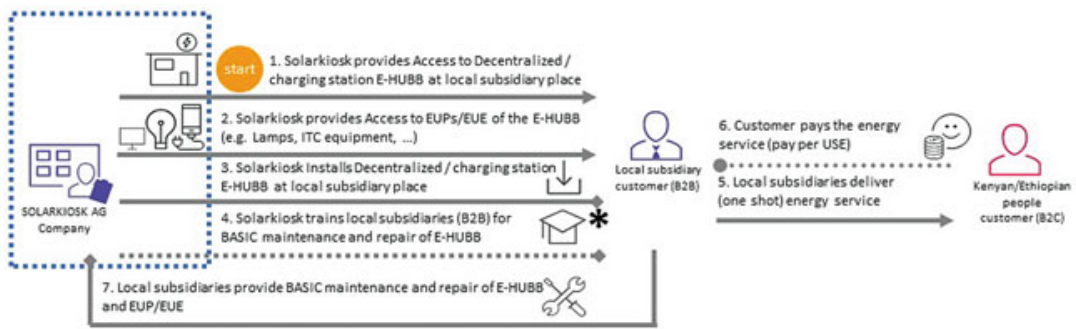

FLOWS LEGEND Information $=n \rightarrow$ Labor $\Longrightarrow$ Finance $\cdots \odot$ Materials $\Rightarrow$

WIN-WIN VALUE: PROVIDER enlarges his/her market towards new (otherwise inaccessible) CUstomers. CUSTOMER (B2B) can access secure energy fostering his/her business viability, while improving his/her competences in maintenance and repair, as well as business management.

Fig. 5 Solarkiosk system map

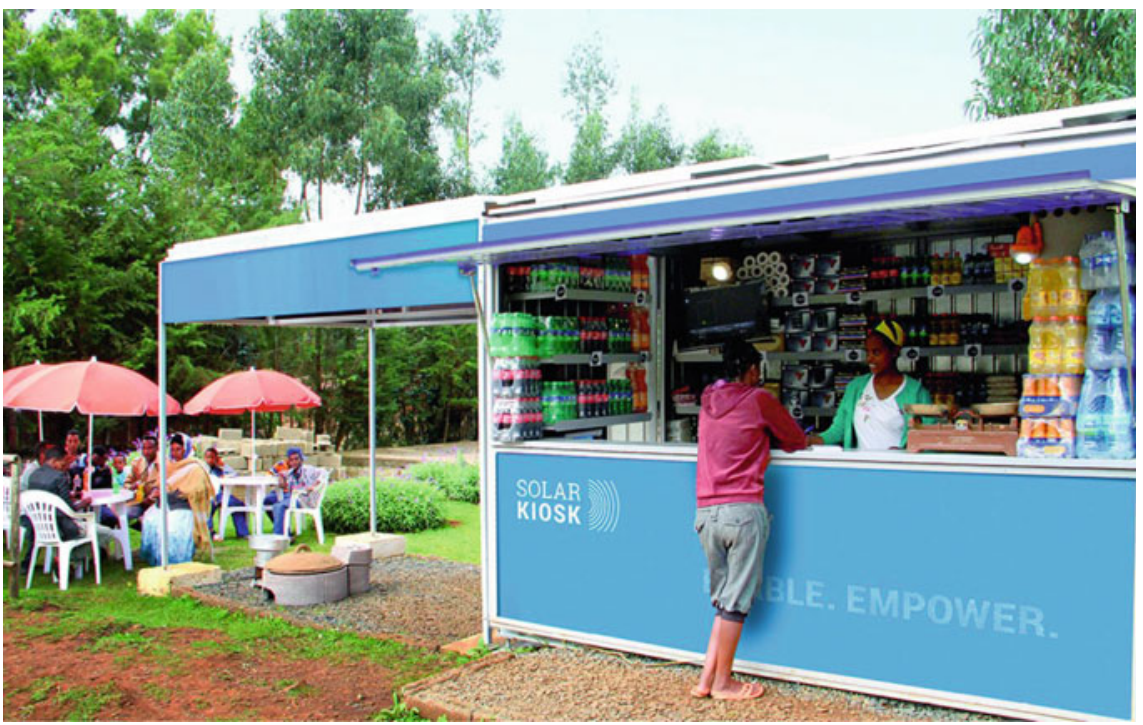

Fig. 6 Solarkiosk Ethiopia, 2011

The new configuration results in reducing the need for transportation of food from outside the city. It also minimizes the use of packaging and storage. Producers and consumers connect with each other, and consumers assume a new role as coproducers who have the opportunity to learn more about local food production, while they get involved in the process of growing food and raising animals. In this way, 
urban farming empowers communities to share knowledge and diversity, keeping alive food traditions and local food heritage.

Several case studies of S.PSS and Distributed Food production (DF) are presented below.

\section{PickYourOwn}

Active since: 2008

Provider/s: Farmers

Customers: Home users (B2C), Commercial Business (B2B)

S.PSS Type: Use-oriented S.PSS

DE configuration: Decentralized Food production

Products: Fruits and vegetables

Services: Use of kitchen and canneries facilities, channel for collaboration, education, consultancy and certified production.

Payments: Pay per period/time or pay per produced unit or each process for the use of kitchen/caning facilities. Pay per product (farm)

\section{Location: USA}

Pick-your-own is an idea for home or commercial users to pick their own fruit from the local farms near them and use them in distributed food production. The website PickYourOwn.org lists farms located all around the country who provide their products to be sold with the pick-your-own concept. On the website there is also a calendar of the harvesting time of different products. The home users or commercial users can pick fresh vegetables and fruits on these farms and produce canned/bottled/packed products using the kitchen/canning facilities that are in shared/community/commercial kitchens and canneries. The users can produce products for their own use as well as to sell or exchange. While most of the facilities are more oriented towards home users, some are oriented towards commercial users. In most sites, they also provide information and education for production in their facilities. Some have licenses that enable users to produce for commercial use. They also function as a hub for users to meet, collaborate and learn from each other. The two common payment methods are pay per period, pay per produced unit/each process or a combination of both. In this case, while the production and consumption of vegetables is distributed, the production also includes the service of fresh food combined with the customer experience of handpicking it. It also reduces the need for packaging and transportation for the producer as well as ensuring a fair price for the produce (Figs. 7 and 8). 


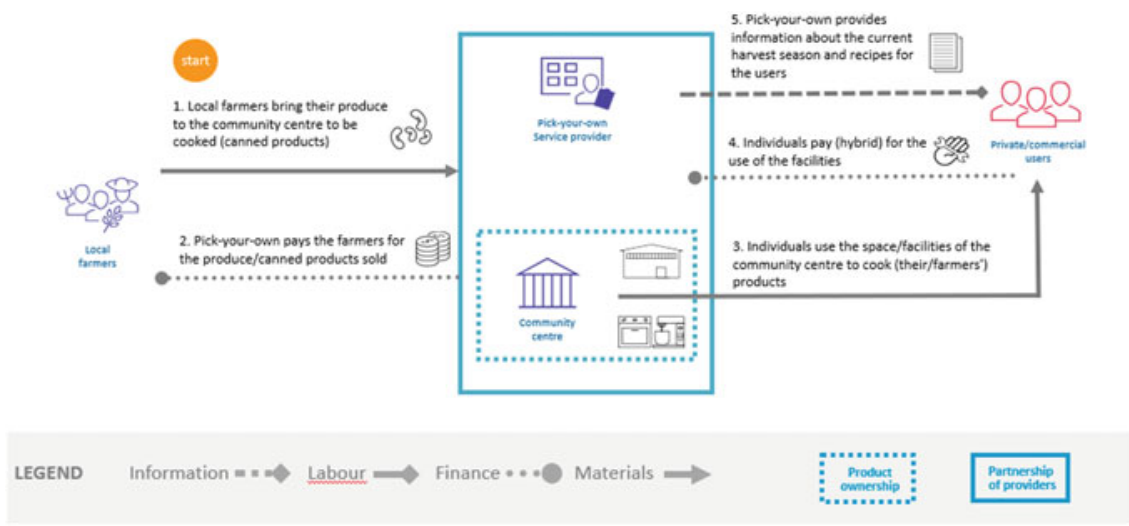

Fig. 7 Pick-your-own system map

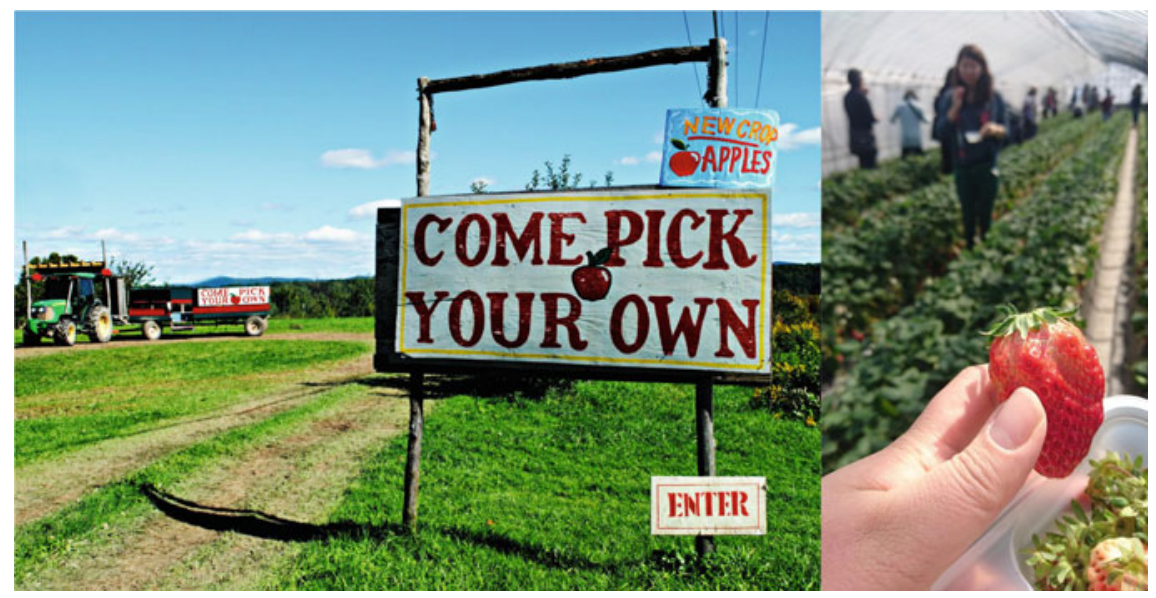

Fig. 8 Example of a Pick Your Own farm, 2008

\section{FoodyBuddy}

Active since: 2017

Provider/s: Home Chefs

Customers: Hungry Individual Consumers

S.PSS Type: Use-oriented S.PSS 


\section{DE configuration: Decentralized Food production}

\section{Products: Fresh Cooked Meals}

Services: Aggregator platform connecting home-based chefs to customers

Payments: Pay per product delivered

\section{Location: India}

Foodybuddy is a neighbour food network that connects home-based chefs to customers at a hyper-local level. The app allows home chefs to decide upon the menu, number of portions, days of sale, timings and pricing of meals, allowing for flexible income generation. The consumer has the advantage of viewing a daily menu of food on offer in their neighbourhood or apartment complex and communicate with the seller on the app.

Since this system works at a hyper-local level, it eliminates the need for transportation. The food is either delivered by the seller or picked up by the consumer. This also allows the seller and the customer to interact personally and develop connections within the neighbourhood. There is an opportunity to connect this service with existing delivery services if it is so required, as an example of a networked distributed system (Fig. 9).
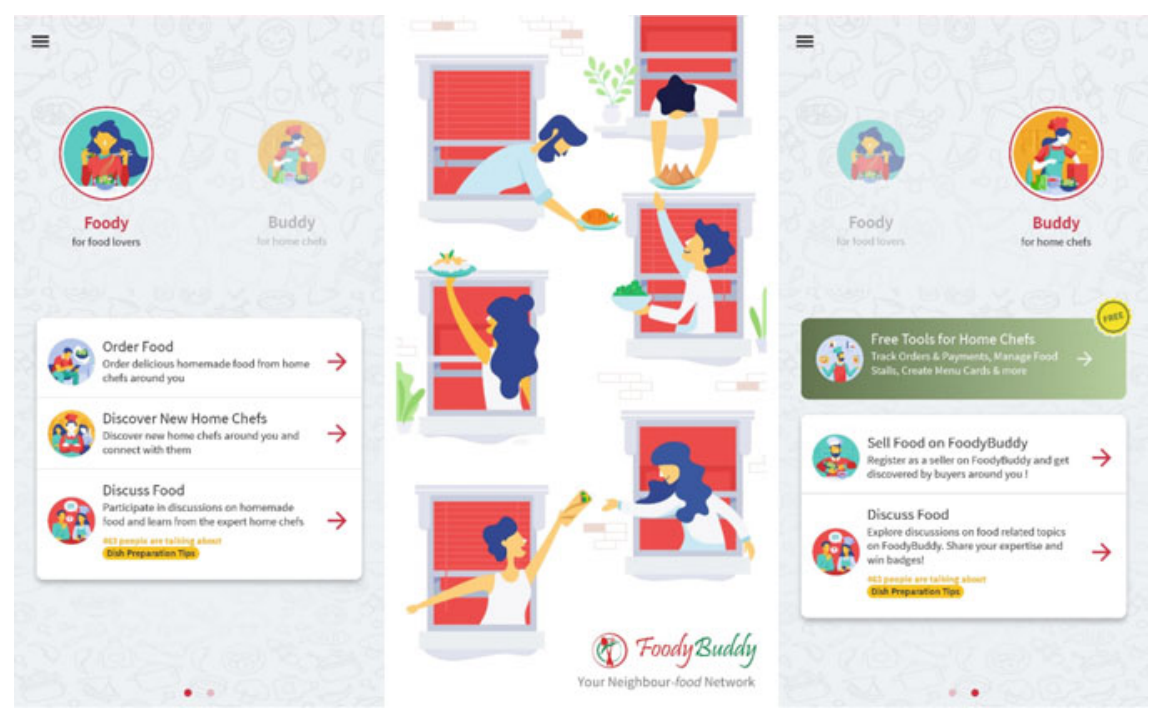

Fig. 9 Service onboarding on the Foodybuddy App. (Source Foodybuddy App) 


\subsection{S.PSS and Distributed Water Management (DW)}

Water management is an area that is increasingly witnessing the dangers of the failure of excessive centralization. With the development of regional scale systems of water management like large dams and reservoirs-primarily for agriculture and power generation - there is evidence of increasing negative impacts on ecosystems [1].

Decentralized solutions for water collection, storage, treatment and use are being revived from traditional systems or developed as new solutions to cater to the needs of vast populations underserved by centralized water management projects. In many parts of the world, limited access to fresh water is also becoming an issue of political contention which disenfranchises vast numbers of people from the process of water management and access. In this scenario, provision of clean water as a service has great potential for developing distributed models of management and access that also empowers communities to be self-sufficient and fosters community-based income generation models. There are organizations that work with community-based catchment management, water storage and treatment.

Several examples of organizations that provide potable water to underserved communities in an S.PSS and Distributed Water management (DW) are presented below.

\section{Piramal Sarvajal}

Provider/s: Piramal Sarvajal with local franchisees

Customers: Underserved rural and urban communities

S.PSS Type: Use-oriented S.PSS

DE configuration: Decentralized Water Management Network

Products: Water ATMs, Water purifiers, Water Quality Monitoring Units

Services: Community awareness and training, centralized water quality monitoring, water delivery system

Payments: Pay per use

\section{Location: India}

Piramal Sarvajal sets up community-level solutions that are locally operated but centrally managed on a market-based pay-per-use system. The last-mile operational accountability is ensured by developing and deploying remotely monitored and controlled drinking water purification systems. Piramal Sarvajal's other product is the Water ATM: a solar-powered, cloud-connected, smart card-based automatic water vending machine. 
While the water purification and delivery systems follow a pay-per-use S.PSS model with an emphasis on socio-ethical and economic sustainability, the distributed system of water purification also allows for developing distributed economies through community-based franchisees (Fig. 10).

\section{Ecosoftt and Gram Vikas}

Provider/s: Ecosoftt and Gram Vikas (Partner NGO)

Customers: People from villages without access to clean water

S.PSS Type: Use-oriented S.PSS

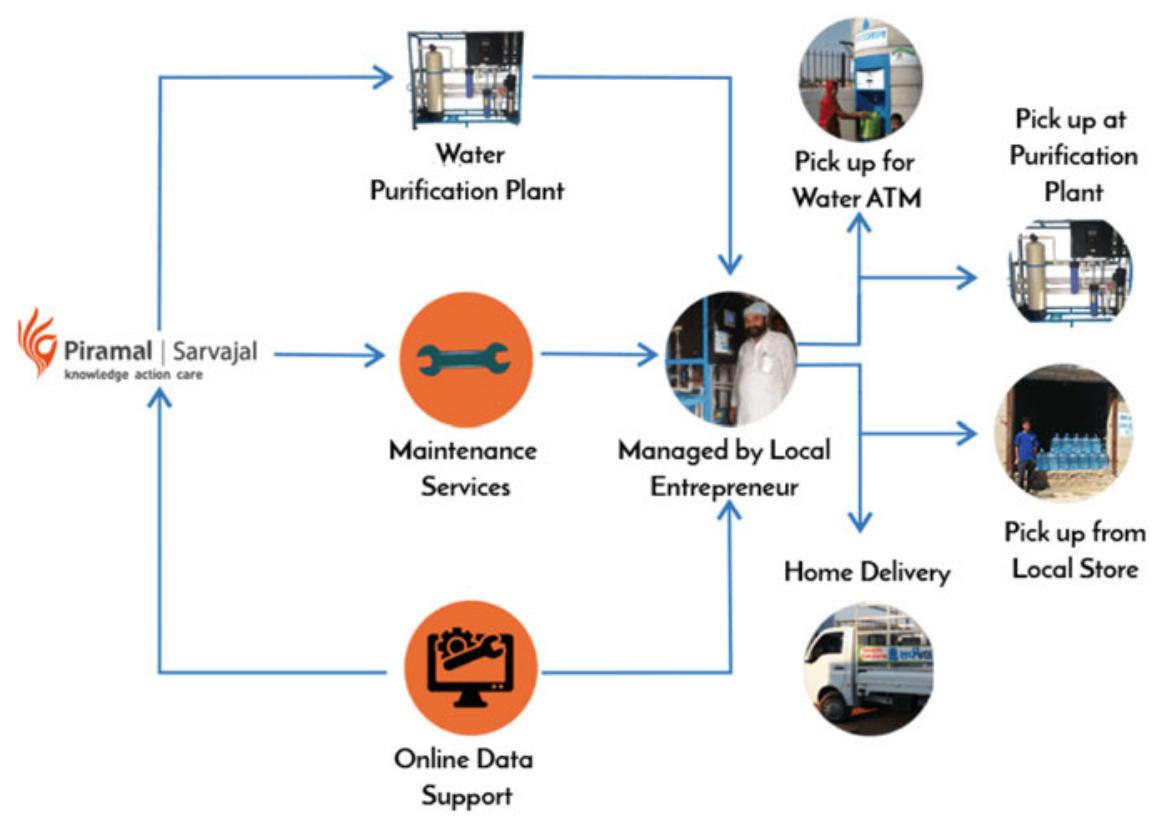

Fig. 10 System map of Piramal Sarvajal. (Source Piramal Sarvajal) 
Fig. 11 Decentralized clean water systems for villages

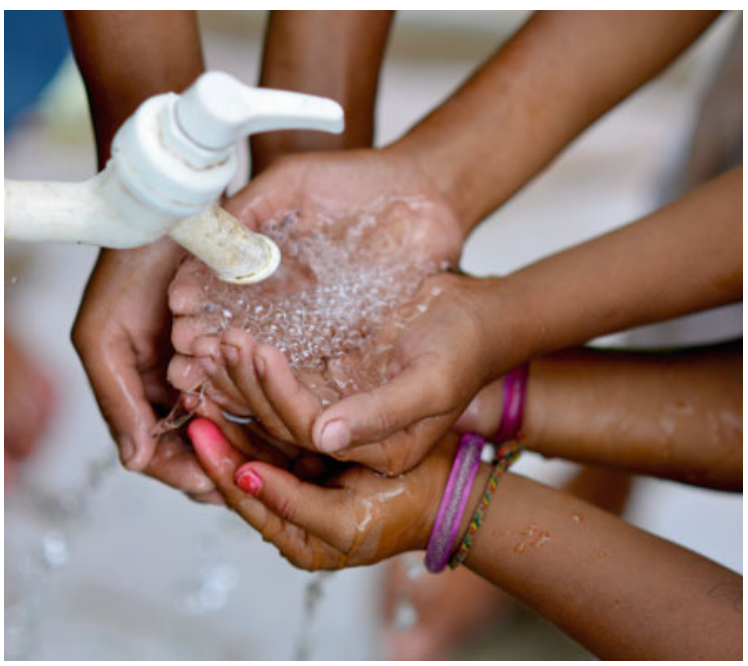

DE configuration: Decentralized Water Management

Products: Equipment to build infrastructure

Services: Access to drinking water, toilets, bathing rooms and wastewater management systems

Payments: Pay per use

\section{Location: India}

Ecosoft in collaboration with Gram Vikas (an NGO) provides equipment + training for local users to build infrastructure to take water from underground and provide decentralized access to clean water in the village. The package consists of access to drinking water, toilets, bathing rooms and a wastewater management system. There is no investment cost for the local community; they pay to Ecosoft according to the amount of water they consume. The package also includes training for maintenance and providing equipment in case of replacement needed (Fig. 11).

\subsection{S.PSS and Distributed Manufacturing (DM)}

Current manufacturing and supply chains have become extremely efficient global systems that draw labour, material, production and assembly from centres around the world. These supply chains have been honed to function at maximum efficiency. However, it is also notable that this efficiency comes at the cost of redundancy and 
resilience. Global events like pandemics are proving that there is a dire need for developing more resilient and localized systems of production and distribution.

As mentioned in Chap. 2, three key features of digital manufacturing have been identified as:

- Localization of manufacturing units;

- Application of physical and digital technologies;

- Customer orientation.

Distributed manufacturing allows more people to develop local livelihood opportunities that can contribute towards building economic sustainability. A movement away from extractive global manufacturing processes marks a potential to develop ecological and socio-ethical sustainability in local communities.

Several case studies of S.PSS and Distributed Manufacturing (DM) are presented below.

\section{StrataSys Leasing}

Active since: 2011

Provider/s: StrataSys

Customers: Small and large enterprises, makers, designers, engineers (B2B)

S.PSS Type: Use-oriented and Result-Oriented S.PSS

DE configuration: Decentralized Manufactoring

Products: 3D printers, start-up supplies, support removal system, cleaning agent

Services: Optional services (system operation, inhouse support, education, project implementation, consulting)

Payments: Pay per period (fixed cost)

Location: USA and Israel (headquarters), Canada, Brazil, Mexico, Germany, Japan, Korea, China, Singapore, India

StrataSys manufactures 3D printers and offers 3D production systems for officebased additive manufacturing, rapid prototyping and direct digital manufacturing solutions. The company offers leasing service of some models of their manufactured commercial 3D printers and bundled 3D-printer packages in the United States. Besides the printer, the 3D Print Packs include start-up supplies, a supportremoval system and cleaning agent. StrataSys also provides various separate services such as system operation, in-house support, education, project implementation and consulting (Figs. 12 and 13). 


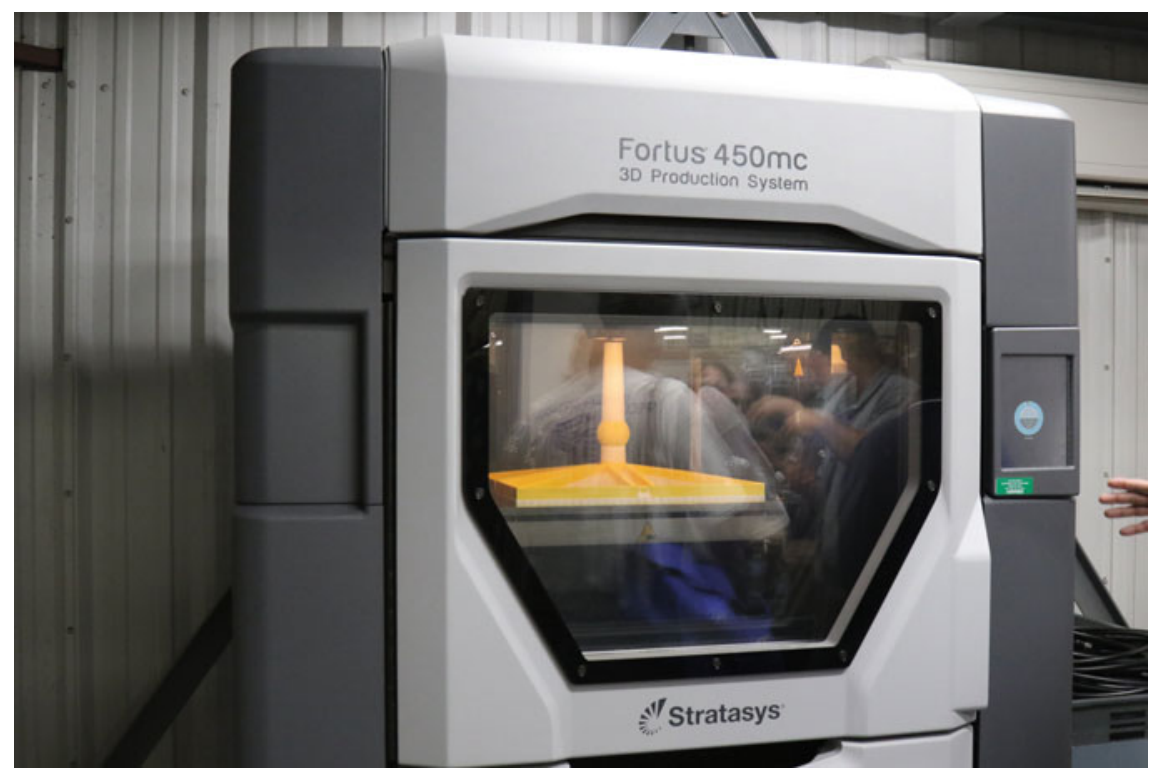

Fig. 12 StrataSys direct digital manufacturing solutions leasing, 2012

STRATASYS

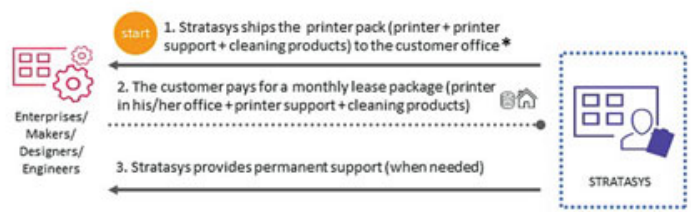

FLOWS LEGEND Information $=n-\$$ Labor Finance .... Materials

Fig. 13 StrataSys system map

\section{Industree Foundation}

Active since: 2000

\section{Provider/s: Industree Foundation}


Customers: Farmers, Artisans

S.PSS Type: Result Oriented

DE configuration: Decentralized Manufacturing

Products: Sustainable Producer Owned Enterprises

Services: Training enterprise leaders in business management, soft skills and hard skills, connecting to academia and designers, creating access to capital and markets, providing digital connectivity

\section{Location: India, Ethiopia}

The Industree foundation organizes rural communities in a distributed value chain yet integrated through an aggregated national level marketing and sourcing enterprise, with whom producer-owned enterprises have the choice to interact for some or all their transactions. The company holistically tackles the root causes of poverty by creating an ownership-based, organized creative manufacturing ecosystem for microentrepreneurs, most of whom are women. Distributed Design and Manufacturing that is equitable and sustainable can be viable only if there is an enabling ecosystem of support. Industree works to co-create an enabling platform using its $6 \mathrm{C}$ principle:

1. Construct: Business model innovation through producer ownership and inclusive entrepreneurship. Producer members earn through fair wages for production and shared profits from production and marketing. Aggregation for viability in material sourcing, professional management, productivity, access to market and capital.

2. Capacity: Training encompasses a grassroots business academy that trains producers and micro-enterprise leaders, paraprofessionals who work in the unit as professional support and service, and enterprise leadership. Training of professionals and enterprise leaders for broad handholding for replication and adaptation beyond Industree.

3. Create: Co-creation of design by professional designers who are part of the professional management group, alongside master artisans, designers would also be part of the professional management team. The efforts of the inhouse team will be bolstered by students and academic institutions from the region and beyond convened by Industree.

4. Capital: Creating access to capital through partnerships with Non-Banking Financial Company (NBFC) and working capital pools. A revolving working capital pool will be created along with funds offered through schemes of the Micro, Small and Medium Enterprises (MSME) sector and access to loans from banks based on purchase orders received.

5. Channel: Markets, connecting to markets both $B 2 B$ and $B 2 C$, creating the awareness among buyers through meets and workshops, using brands to connect with 


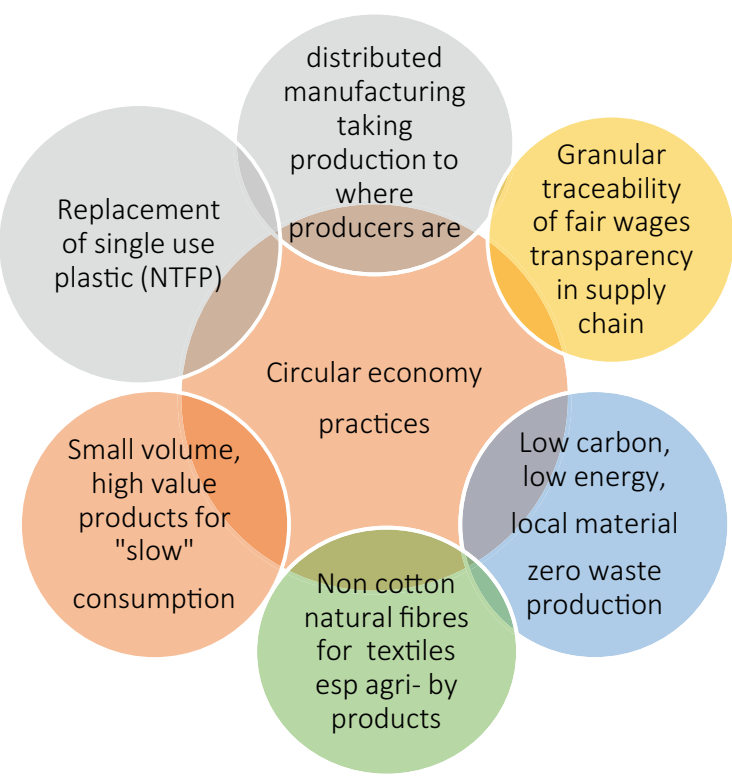

Fig. 14 An example of Circular Economy practices of Industree

brands. Participation in fairs and exhibitions to promote the products, nationally and regionally.

6. Connect: Digital connectivity primarily through mobile applications, through which sustainable enterprises in the creative manufacturing can be supported, serviced, incubated and accelerated (Figs. 14 and 15).

\subsection{S.PSS and Distributed Software (DS)}

While the internet began as a decentralized network of servers accessed by a network of users, there has now been a shift towards an increasingly centralized net through intermediaries like Google and Facebook whose servers handle a significant portion of all data on the internet. This has led to concerns over individual and organizational privacy, data protection and data ownership and agency. There is also a growing realization that these intermediaries have disproportionate control over information flows. Since online media now encompass critical sectors like finance, social networking and business, there are emerging alternatives that seek to develop networks of distributed and localized data storage and application embedded in communities rather than global corporates.

The case studies of S.PSS and Distributed Software (DS) presented below demonstrate a movement towards community-based and community-led online services. However, it is to be noted that although distributed software and in particular the 


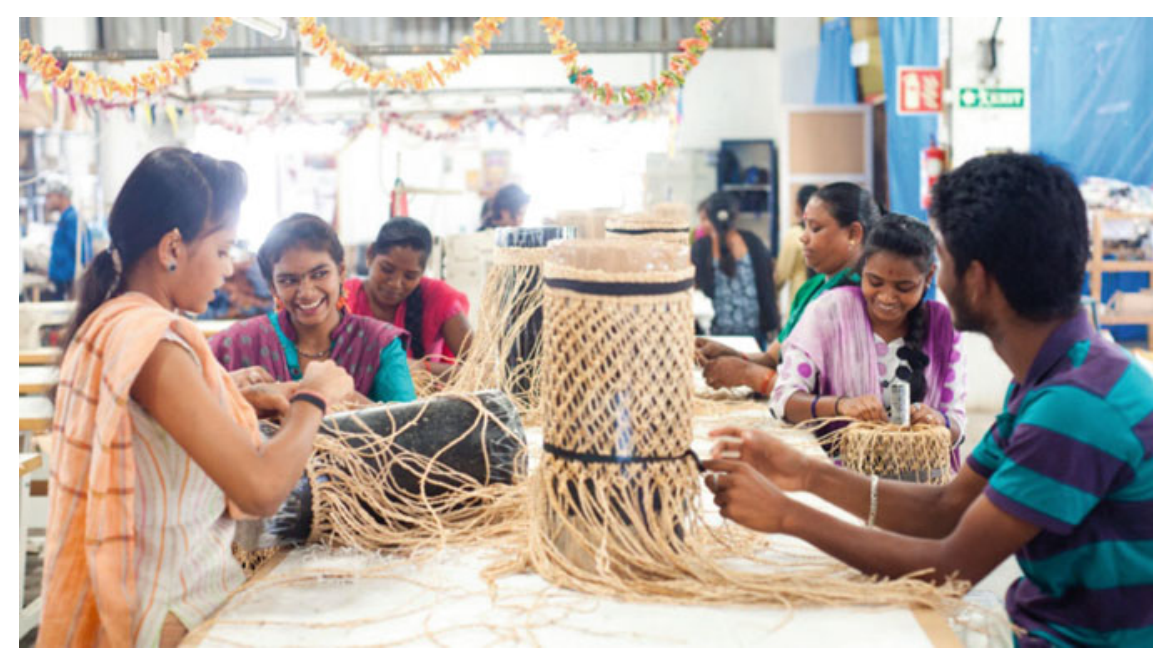

Fig. 15 Bangalore GreenKraft—one of the enterprises set up by Industree

case studies chosen show potential opportunities for developing S.PSS models, they have not yet actively incorporated it into their current form.

\section{Secure Scuttlebutt}

Active since: 2014

\section{Provider/s: Secure Scuttlebutt}

Customers: Community

S.PSS Type: Result-Oriented

DE configuration: Decentralized Software

Products: Offline Friendly Secure Gossip Protocol

Services: Data Ownership, End to End encryption, Agency over interaction

Payments:-

\section{Location: Worldwide (origin New Zealand)}

Secure Scuttlebutt is a localized but distributed social network that works with a peer-to-peer mesh network where user data is stored locally on user devices rather than a centralized server [7]. The data is exchanged between devices through data replication on a shared WiFi or local area network or even with a USB stick. It is 
also possible to connect to the network using public servers called "Pubs". The intent of Secure Scuttlebutt is to eliminate the need for connection to centralized servers while still having the network intact through a localized community of devices. This develops a resilient system that is upheld through the distributed network.

On a voluntary basis, it is possible for users to engage monetarily using the Secure Scuttlebutt Consortium. As an S.PSS model, in exchange for a voluntary donation, the developers are able to provide an opportunity for an alternate social media network that protects user data and allows the user to choose terms of engagement with the network. It connects people who do not have access to a regular internet service and can also be used in emergency situations.

\section{Holochain}

Active since: 2006

Provider/s: Holochain is a technology that can be used by multiple providers

Customers: Communities of users and developers

S.PSS Type: Product- or Use-oriented S.PSS (depending on the application)

DE conf.: Distributed Software

Products: HoloPort device for hosting (optional)

Services: Framework and protocol for app development

Payments: Hosts are paid in crypto; Holo takes a percentage transaction fee

\section{Location: Worldwide}

Holochain is a framework for building distributed peer to peer applications that is based on a shift from data centric computing of the Blockchain to an agent centric model. Holochain is a way of building and running applications on the user's own devices and without using an intermediary server. Users within a community that have spare computing capacity on their devices can host the applications of others. In exchange for this, the contributor gets paid in Holo Fuel, a crypto-currency that can be used to buy applications or hosting services within the community. Another characteristic of Holochain's agent-centric approach is that the users determine the terms of engagement within their own communities and this cannot be disrupted by others.

As a distributed computing system, parallels can be drawn with a two-way power grid, except here computing capacity is shared by users. It is possible to envision ways in which this peer-to-peer sharing system can allow users to build a sharing ecosystem of online and offline services in future (Fig. 16). 

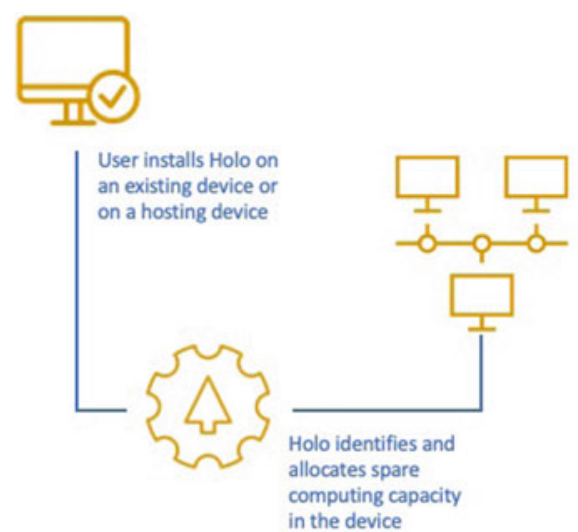

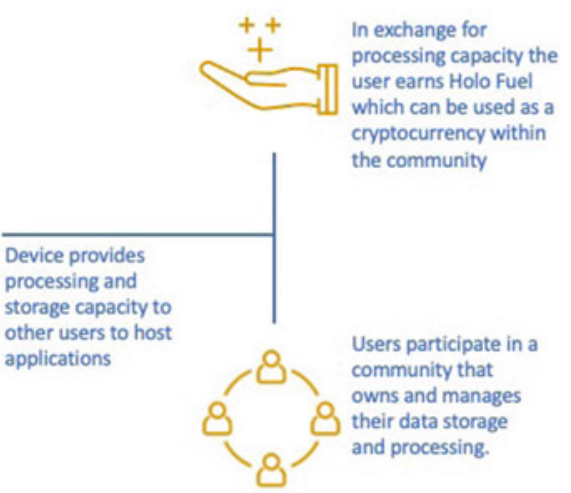

Fig. 16 Holochain system (Adapted from [5])

\section{Linux}

Active since: 1991

Provider/s: Community of developers

Customers: Community

S.PSS Type: Use-oriented S.PSS

DE conf.: Distributed Software

\section{Products: Computer code}

Services: -

Payments: Free

\section{Location: Worldwide}

Linux is an open-source operating system that is used in smartphones, personal computers, netbooks, supercomputers, servers, embedded devices, home appliances, cars and so on. The source code can be used, modified and distributed by anyone under the GNU General Public License, with the condition that whoever distributes software using a source code under the GNU license must make the original as well as the modified source code available under the same terms. Thus it can be said that Linux is software produced by a network of developers e.g. small-scale producers that are connected with each other locally and globally (Fig. 17). 


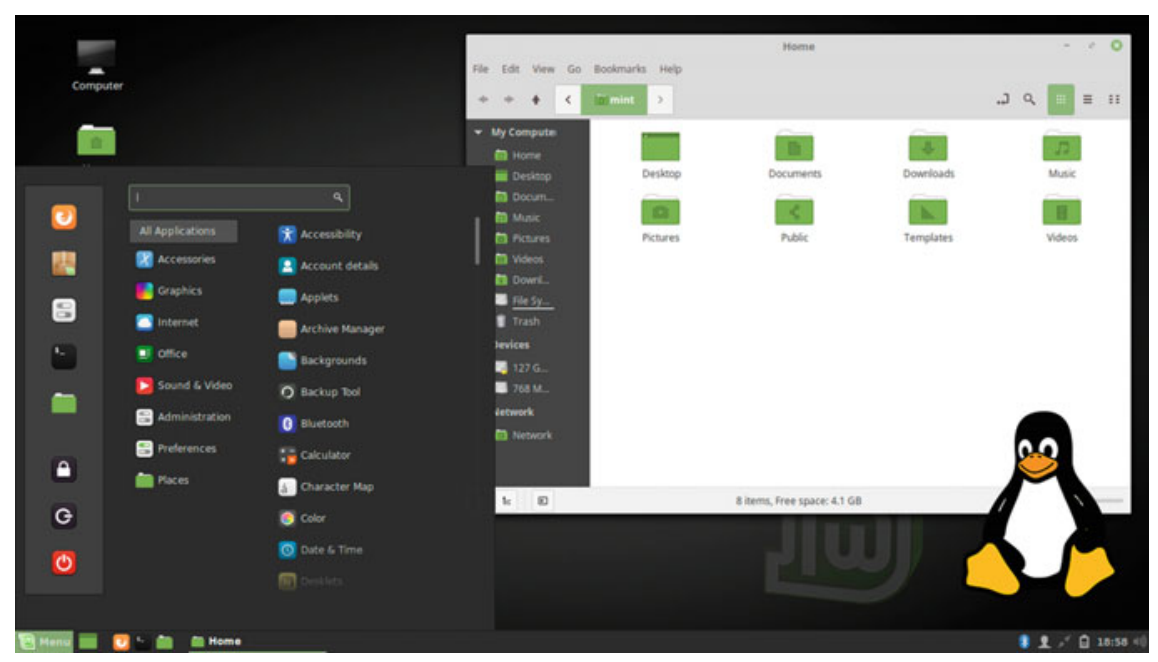

Fig. 17 Examples of Linux application

\subsection{S.PSS and Distributed Knowledge (DK)}

Internet and web technology has revolutionized learning by providing a vast amount of learning resources across disciplines that are easily accessible and very affordable, often only at the cost of the internet service. One of the main ways in which this has influenced education in universities and schools is bringing ways of learning, thinking and doing to the forefront since it is now fairly common to be able to pick up skills through online resources. There is also a shift towards self-directed learning where students decide which subjects and skills are most appropriate to support their own goals and interests.

A number of online learning platforms like $\mathrm{EdX}$ and Coursera provide online courses from universities across the world and in a range of subjects that can be freely accessed by anyone with internet access. Additionally, on passing a course, it is also possible to pay a nominal fee for receiving a certificate from the respective university.

There are also attempts to draw a link between online learning communities and physical communities in distributed knowledge production and application. The case study of S.PSS and Distributed Knowledge (DK) in this section illustrates one such attempt. 


\title{
Project DEFY (Design Education for Yourself)
}

\author{
Provider/s: Project DEFY
}

Customers: Community

S.PSS Type: Use-oriented S.PSS

DE configuration: Decentralized Knowledge

Products: Nooks

Services: Induction Program for new learners

Payments: Income generation through innovation and projects

Location: India, Uganda, Rwanda, Zimbabwe

\section{Location: India}

Project DEFY sets up self-designed learning centres or 'Nooks' across marginalized communities in India and Africa (Uganda, Rwanda, Zimbabwe). Nooks are freefor-all 'schools without teachers' that provide everyone in the local community with access to technology, tools, resources and information to design their own education. As such, Nooks are a primary example of distributed education design where learning is decentralized, contextualized, localized and individualized.

This process is supported through a 45-day long induction programme for new learners in which they get exposed to new areas of skills and learning through handson practice as well as through fostering and providing a safe, inclusive space for meaningful conversations to take place among the Nook community. At the end of the induction programme, the learners are enabled (individually or in groups) to identify and write down their own, individual goals and help to break them down into concrete, hands-on projects they pursue in order to achieve their goals.

As opposed to schools and colleges where decision-making follows an authoritarian top-down approach, Nooks are managed by the community members themselves. This includes administrative decisions such as the opening times of the Nook, the responsibility for a monthly resources budget, as well as —of course-being in charge of the learning process itself.

Whereas in schools and colleges the learners are separated from the means and resources of learning-having their relations to those means mediated, appropriated, circumscribed and severed by teachers, textbooks, curriculum, etc.-Nook learners are enabled to take control of and directly own the means and resources 


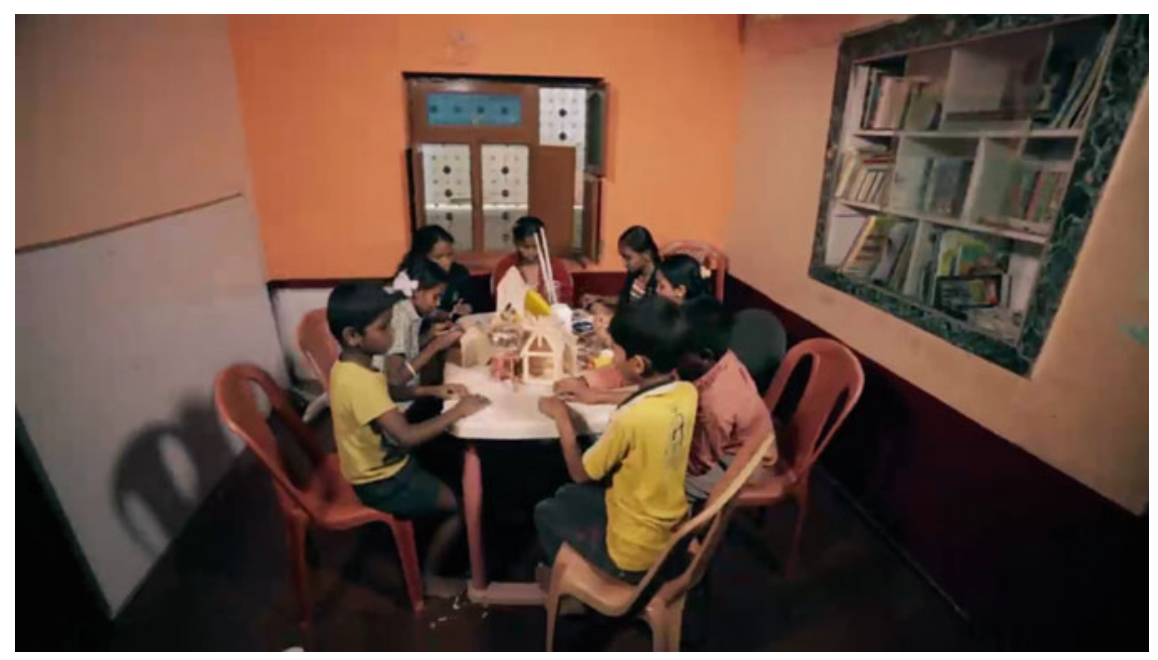

Fig. 18 Nook enabled by Project DEFY (Source: Project DEFY)

of learning. Importantly, Nooks do this on a scale and cost that fits within the economy of even low-income communities. In the long run, the Nooks aim to become self-sufficient and completely community-run by capitalizing on the creations, innovations, products and skills that emerge out of them (Fig. 18).

\subsection{S.PSS and Distributed Design (DD)}

The complex nature of contemporary design problems has led towards an increasingly collaborative and heterogeneous approach to knowledge production and design. There is a recognized need for bringing together experts and stakeholders across disciplines to fully understand the nature of dependencies of a system and to then design appropriate systemic solutions [4, 12].

A number of design platforms and collectives have emerged over the last decade. Some are along the lines of an Uber model, where designers, manufacturers and suppliers are connected with customers to enable distributed design service. In disciplines like architecture and interior design, for example, multiple companies have emerged which provide end-to-end services of design, fabrication, installation and finish with additional options such as home products and maintenance services using distributed networks of local businesses. Other distributed design models such as Local Motors are more topical and specific. 
Several case studies of S.PSS and Distributed Design (DD) are presented below.

\section{Quirky}

Active since: 2009

Provider/s: Quirky (platform, online tool and connection between members and manufacturers), Partners, e.g. General Electric, PepsiCo, Mattel (manufacturing)

Customers: Designers, inventors, individuals with specific skills

S.PSS Type: Result-oriented S.PSS

DE configuration: Distributed Design

Products: -

Services: Provides a network of skilled users and access to product creation enterprises

Payments: Free (use of the platform)

Location: New York City.

Quirky is an invention platform that connects inventors with users who have other skills for developing the idea and with companies specialized in a specific product category for manufacturing. The offer is therefore access to complete product creation. Quirky's business model pays designers part of the profits that their products yield. The users do not need to pay for using the platform. The users can submit their ideas and connect with others to make a team for collaboration. Once the developed idea is accepted by Quirky through a voting system by the Quirky community at Eval (Quirky's live weekly product evaluation), it is pitched to the manufacturers. If it is manufactured, Quirky shares the profit with the team members according to their influence evaluated by a point system on the Quirky platform (Fig. 19).

\section{Local Motors}

Active since: 2007

Provider/s: Enthusiasts, hobbyist innovators, designers, engineers, fabricators and other professionals

Customers: Designers, inventors, individuals with specific skills S.PSS Type: Hybrid of use-oriented and product-oriented S.PSS

DE configuration: Distributed D and Decentralized Manufactoring

Products: Motor Vehicles (rally cars, motorcycles, electric bicycles, tricycles, children's ride-on toy cars, radio-controlled model cars and skateboards) 


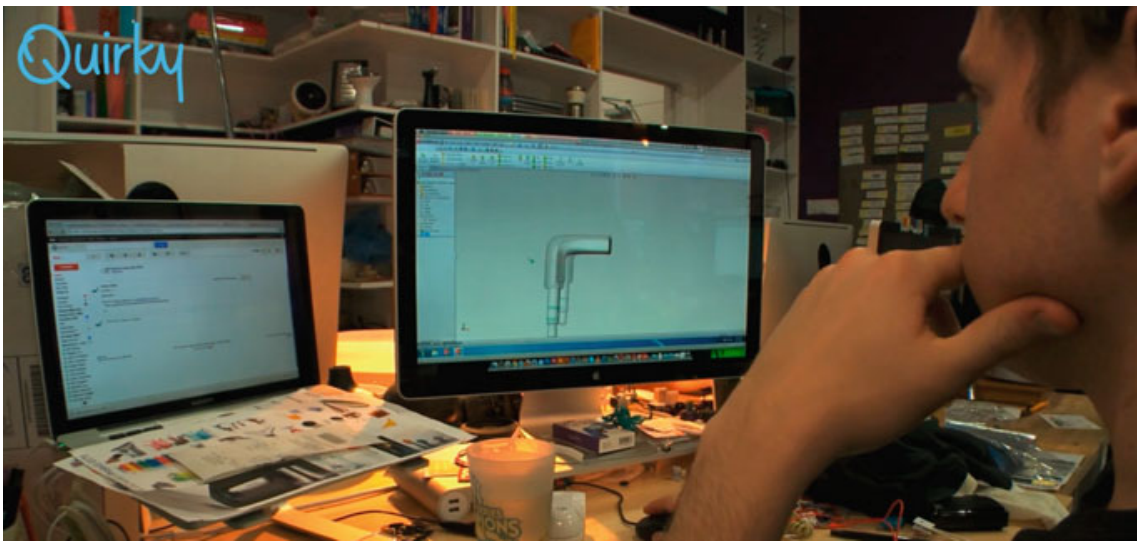

Fig. 19 Quirky invention platform, 2009

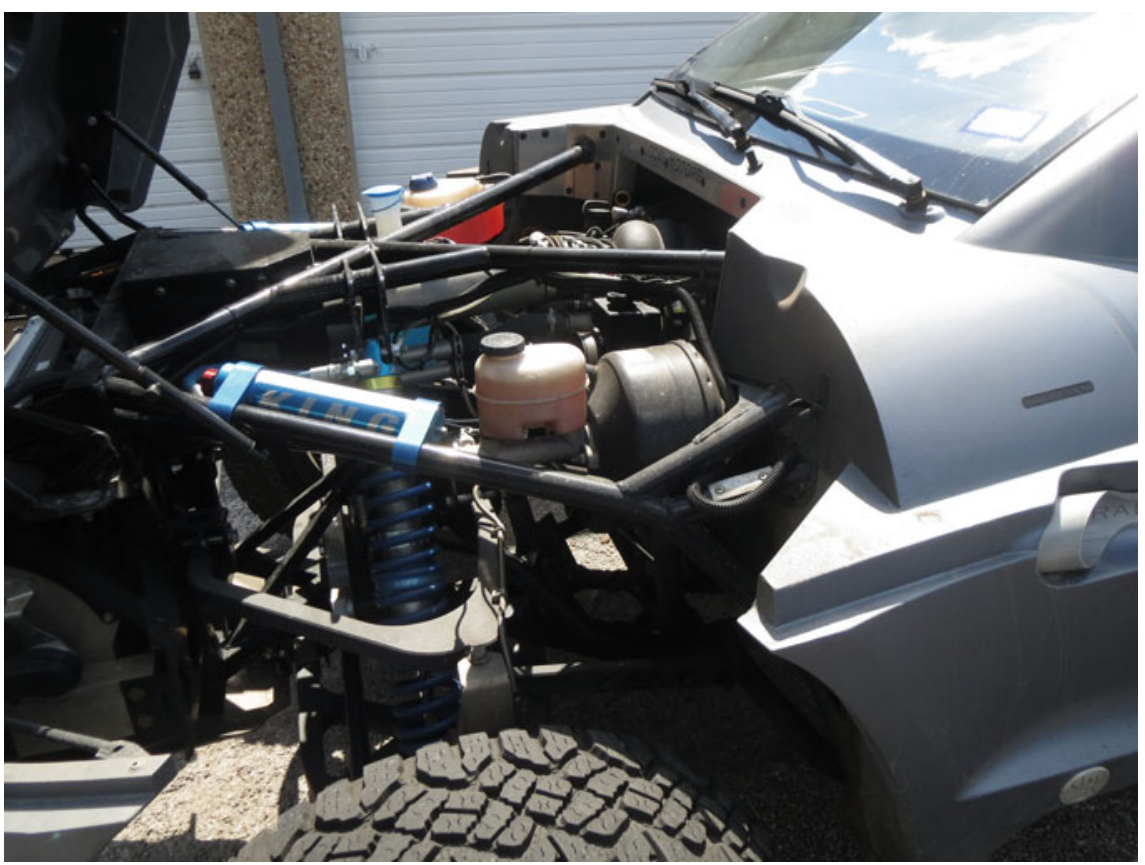

Fig. 20 Local Motors vehicle manufacturing company, 2007 
Services: Management of the global network of microfactories and the co-creation community

Payments: Free (users get even paid in case of revenue for their contribution)

\section{Location: USA}

Local Motors (LM) is a motor vehicle manufacturing company focused on lowvolume manufacturing of open-source motor vehicle designs using multiple microfactories. Their products include the Rally Fighter automobile and Racer motorcycle, various electric bicycles, tricycles, children's ride-on toy cars, radio-controlled model cars and skateboards. They 3D print some components. Rally Fighters have used cocreation techniques, whereby products are designed cooperatively with end-users, as part of its designing phase. Their website is a community focusing on engine vehicle innovation. The content is co-created by the users of the community who discuss designing, engineering and building innovative engine vehicles (Fig. 20).

\section{S.PSS Applied to DE: A Scenario}

Envisioning the coupling of the two offer models, S.PSS and DE, some further considerations highlighting some of their evident characteristics could be given.

First of all, as far as we have diverse types of DE (Distributed energy Generation (DG), Distributed Manufacturing (DM), Distributed production of Food (DF), Distributed Water management (DW), Distributed production of Software (DS), Distributed production of Information/knowledge (DI), Distributed Design (DD) and the 3 main types of S.PSS (Product-oriented S.PSS, Result-oriented S.PSS; Use-oriented S.PSS), it is clear that a set of diverse combinations of these could emerge in principle.

Secondly, another possible main variable is the type of customer or user, i.e. whether a B2B offer, B2C offer, p2p non-market, and so on.

Furthermore, as far as the hardware of each DE and who is producing it is a key characteristic (because in an S.PSS offer model she/he is the one that has the economic interest to redesign it with a low environmental impact), it is of key interest to identify the hardware for each type of DE.

Finally, other characteristics worth highlighting are related to the DE structure types, i.e. they could be Distributed or Decentralized and each of those could be stand-alone or network-structured.

In relation to those variables and their possible sustainable combinations, a Sustainable Design-Orienting Scenario (SDOS) for Sustainable Product-Service System (S.PSS) applied to Distributed Economies (DE) in low and middle-income (all) contexts has been designed ${ }^{1}$ to provide a new vision of sustainable production $\&$ consumption systems.

\footnotetext{
${ }^{1}$ The scenario design process emerged from a case study analysis of S.PSS applied to DE (best practices), as well as an idea generation workshop focused on S.PSS applied to DE using the SDO toolkit (www.lens-international.org). The scenario presented here is an update of a scenario
} 


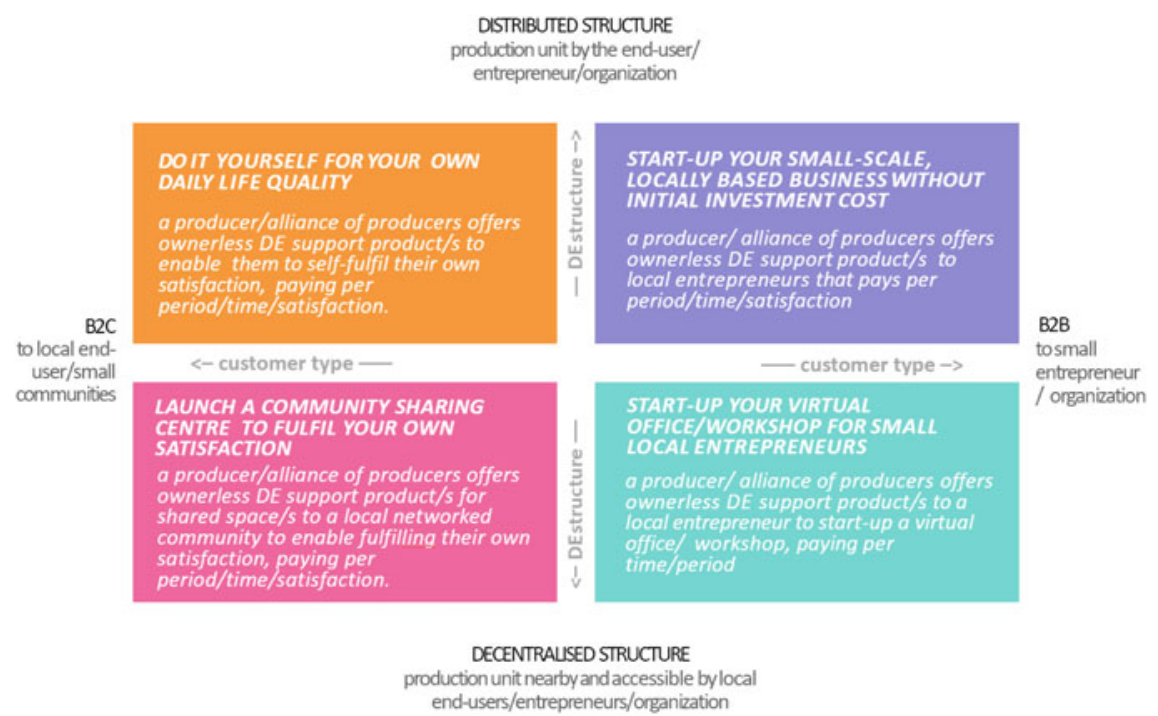

Fig. 21 The Sustainability Design-Orienting Scenario for S.PSS applied to DE

The Scenario is composed of a polarity diagram with 4 visions, for each of the 4 quadrants in the diagram matrix. Each vision represents a Sustainable win-win configuration, combining socio-cultural, organizational and technological factors, fostering solutions with a low environmental impact, a high socio-ethical quality and a high economic value.

The scenario matrix is polarized on the vertical axis by the type of DE structure, distributed or decentralized, and on the horizontal axis by the type of customer/user, B2C (final user or small communities) or B2B (small entrepreneur or small business). The crossing of those polarities produced the following 4 visions, relative to the four quadrants (see Fig. 21) ${ }^{2}$ :

A. [distributed-B2C] DO IT YOURSELF FOR YOUR OWN DAILY WELLBEING: a producer/alliance of producers offers ownerless DE support product/s to enable the end-user to self-fulfil their own satisfaction, paying per unit of period/time/satisfaction.

B. [distributed-B2B] START-UP YOUR SMALL-SCALE, LOCALLY BASED BUSINESS WITHOUT INITIAL INVESTMENT COST: a producer/alliance of producers offers ownerless DE support product/s to local entrepreneurs that pay for unit of period/time/satisfaction.

C. [decentralized-B2C] LAUNCH A COMMUNITY SHARING CENTRE TO FULFIL DAILY LIFE SATISFACTION: a producer/alliance of producers

developed by Cenk Basbolat for his degree thesis at the School of Design of Politecnico di Milano, tutored by Carlo Vezzoli.

${ }^{2} \mathrm{~A}$ set of videos presenting the visions of the scenario as well as their possible options are available at http://lens-europe.eu/tools/view/2 
offers ownerless DE support product/s for shared space/s to a local networked community to enable fulfilling their own satisfaction, paying per period/time/satisfaction.

D. [decentralized-B2B] START-UP AS SMALL, LOCAL ENTREPRENEURS WITH VIRTUAL OFFICE/WORKSHOP: a producer/alliance of producers offers ownerless space for an office and/or workshop equipped with DE support product/s to a local entrepreneur to start-up its business, paying per unit of time/period.

To illustrate the scenario, we now describe one example (case study) per each of the visions.

\section{A. Do-it-yourself for your own daily life quality: example}

Qurrent: The company teaches customers how to produce and manage renewable energy, allowing them to organize the exchange of energy in small local networks. Qurrent offers Solar Home Systems (SHS) composed of photovoltaic panels (and related components) and three core products: Qbox, Qmunity website, Qserver. Specifically, the Qbox measures all production and consumption of electricity and makes it possible to share capacities with the neighbourhood.

\section{B. Start-up your small-scale, locally-based business without initial investment} cost: example

SELCO: With the support of government funds and bank loans, SELCO facilitates and enables financing options for rural entrepreneurs to set up solar powered enterprises like photocopying and printing kiosks, tailoring units and mechanized cattle milking units in underserved areas. This generates sustainable livelihood options and offers access to services for the community.

\section{Launch a community sharing centre to fulfil daily life satisfaction: example}

Helsinki Metropolitan Area Reuse Centre: The Reuse Centre sells donated second-hand goods and building and hobby materials in their retail outlets, which are located in many locations in Helsinki, Finland. The organization makes it easier for customers to reuse and recycle by offering transportation services and leasing pull-trailers for a fee, and customers can borrow a cargobike for free if they purchase something from one of the shops. The Reuse Centre also provides educational workshops on recycling and the environment to children. 
D. Start-up a virtual office/workshop for small, local entrepreneurs: example

Maker Station: Maker Station is a large makerspace, workshop and co-working space that provides access to industrial tools and equipment, studio space and storage space for artists, artisans, designers and small producers on a membership basis in Cape Town, South Africa. It also links artists, artisans and designers with projects and companies needing their skills and labour, and it provides technology workshops for hobbyists and marginalized children. The equipment and tools it provides include milling machines and lathes, laser cutters, CNC vinyl cutters, $3 D$ printers, electronics stations, sheet metal equipment, welding equipment, woodworking equipment, hand tools and sewing machines.

\section{Exploring opportunities within the S.PSS applied to DE Scenario}

Within the SPSS applied to DE Scenario, the following strategies (and guidelines) have been identified ${ }^{3}$ as potential diversification of proposals within each of the visions:

- Complement DE hardware offer with Life Cycle services

- Offer ownerless DE systems as enabling platform

- Offer ownerless DE systems with full services

- Optimize stakeholders' configuration

- Delink payment from hardware purchases and resource consumption

- Optimize DE structure.

\section{Complement DE hardware offer with Life Cycle services}

- The provider/s complements the offer of the DE system with:

- financial services to support initial investment and eventual maintenance and repairing costs, e.g. micro-credit, crowdfunding, donation to maintain, repair one or more DE hardware/components

- support services for the design and/or installation of its components (e.g. in DG, the micro-generator, the storage, the inverter, the wiring, etc.)

- support services during use, i.e. maintenance, repairing and upgrading of its components

- support services for the end-of life treatment of its components

- support services to enable the customer to either design and produce with their DE hardware, share their DE hardware, sell/provide their production, provide services through their DE hardware.

\footnotetext{
${ }^{3}$ Those presented here are an update of a set of criteria and guidelines developed by Cenk Basbolat for his degree thesis at the School of Design of Politecnico di Milano, tutored by Carlo Vezzoli.
} 
Offer ownerless DE systems as enabling platform

- The provider/s complements an ownerless offer of the DE system with training/information services to enable the customer:

- to design the DE hardware/components

- to maintain, repair one or more DE hardware/components

- to install one or more DE hardware/components

- to upgrade one or more DE hardware/components

- to optimize use of one or more DE hardware/components

- to either design, produce with their DE hardware, share their DE hardware, sell/provide their products, provide services through their DE hardware.

Offer ownerless DE systems with full services

- The provider/s complements an ownerless offer of the DE system with full support services:

- to design the DE hardware/components

- to maintain, repair one or more DE hardware/components

- to install one or more DE hardware/components

- to upgrade one or more DE hardware/components

- to optimize use of one or more DE hardware/components

- to either design, produce with their DE hardware, share their DE hardware, sell/provide their production, provide services through their DE hardware.

Optimize stakeholders' configuration

- Offer the S.PSS to the final user, or a collective, to improve the quality of life or the environment

- Offer the S.PSS to an entrepreneur to enable a business start-up or empower business

- Optimize a stakeholder partnership with vertical integration by combining all complementary components of one single DE type (e.g. in DG, the microgenerator, the storage, the inverter, the wiring, etc.)

- Optimize a stakeholder partnership with horizontal integration (by combining different DE offers as a full package offer)

- Make the DE hardware manufacturer S.PSS offers either alone or in a joint venture with another stakeholder

- Make the DE service provider S.PSS offers either alone or in a joint venture with another stakeholder.

Delink payment from hardware purchases and resource consumption

- Offer pay x period, i.e. the cost is daily/weekly/monthly/yearly fixed

- Offer pay $\mathbf{x}$ time, i.e. the cost is fixed per minutes/seconds of access

- Offer pay $\mathbf{x}$ use/satisfaction unit, i.e. the cost is fixed per product performance (e.g. $\mathrm{km}$ for a vehicle, washing cycles for a washing machine)

- Offer payment based on hybrid pay x period, pay x time, pay x use modalities 
- Offer other economic transactions not based on financial currencies, such as time exchange or direct exchange of goods

- Apply for additional financial support from public administrations/entities.

\section{Optimize DE structure}

- Offer stand-alone DE Product-Service Systems for homes or business sites (especially isolated sites)

- Offer local mini-network connecting DE systems, to enable local production surplus sharing or for enabling shared use of the DE hardware and sharing operations for DE service provision

- Offer decentralized stations, e.g. 3D printing service spot, charging spot, etc., for local communities or decentralized service providers, e.g. a local technician's shop

- Offer decentralized systems to locally supply DE production throughout a mininetwork for homes and/or business sites or a mini-network of service providers

- Offer the DE system with a connection to a worldwide network/main-grid, enabling homes, small business and communities the selling/purchasing of production or for enabling shared use of the DE hardware and/or the shared provision of local services.

\section{Barriers and Trade-Offs to Integrating S.PSS and DE}

Distributed Economies evolve with context and situation to provide different opportunity spaces that can attract new stakeholder configurations. This requires a constant adjustment of the S.PSS model, which can be informed by these changing opportunities. This means that the organization needs to be flexible in allowing possibility for customization according to local needs and context of different network nodes in terms of providing a relevant S.PSS model.

As evidenced by many of the case studies in this chapter, setting up these systems needs long-term engagement with local communities and networks. It must include capacity building, community mobilization and awareness creation at multiple nodes. Bringing together these capabilities and developing networks for learning and sharing the know-how. However, we must have sustained and long-term investment in developing the knowledge and capacity in multiple regional and local centres, and this can be a challenging proposition.

Scaling up of distributed networks again can be challenging, as it requires strategic components to be centralized to optimize resources. Finding the balance between the distributed and centralized components of a product-service system in a distributed economy must include an intelligent business plan backed by policies that support the sustainability of the system.

Distributed Economies may not necessarily provide the most environmentally efficient solution. It is important to assess and balance all three components of sustainability—economic, environmental and socio-ethical—to arrive at the best possible 
model. This would be possible if we had increased access to assessment tools and frameworks as well as modelling technology, all of which today are not very easily available to planners and entrepreneurs at the grassroots level.

\section{References}

1. Beck MW, Claassen AH, Hundt PJ (2012) Environmental and livelihood impacts of dams: common lessons across development gradients that challenge sustainability. Int J River Basin Manag 10(1):73-92

2. Emili S, Ceschin F, Harrison D (2016) Product-service systems applied to distributed renewable energy: a classification system and 15 archetypal models. Energy Sustain Dev 32:71-98

3. Emili S (2017) Designing product-service systems applied to distributed renewable energy in low-income and developing contexts: a strategic design toolkit. PhD Thesis, Brunel University London

4. Giaccardi E, Fischer G (2008) Creativity and evolution: a metadesign perspective. Digital Creativity 19(1):19-32

5. Marin F (2018, March 8). Fixing the internet: how holochain wants to change the way we interact. Ouishare Magazine. https//www.ouishare.net/article/fixing-the-internet-howholochain-wants-to-change-the-way-we-interact. Accessed 22 June 2020

6. Reinders A, Diehl JC, Brezet H (2012) The power of design: product-innovation in sustainable energy technologies. Wiley, London, UK

7. Tarr D, Lavoie E, Meyer A, Tschudin C (2019) Secure Scuttlebutt: an identity-centric protocol for subjective and decentralized applications. In: Proceedings of the 6th ACM conference on information-centric networking, pp 1-11

8. Vasantha GVA, Roy R, Lelah A, Brissaud D (2012) A review of product-service systems design methodologies. J Eng Des 23(9):635-659

9. Vezzoli C, Ceschin F, Diehl JC (2015) The goal of sustainable energy for all. J Clean Prod 97:134-136

10. Vezzoli C, Ceschin F, Osanjo L, M'Rithaa MK, Moalosi R, Nakazibwe V, Diehl JC (2018) Designing sustainable energy for all: sustainable product-service system design applied to distributed renewable energy. Springer, London

11. Vezzoli C, Kohtala C, Srinivasan A, Diehl JC, Fusakul M, Xin L, Sateesh D (2014) Productservice system design for sustainability. Greenleaf, Sheffield

12. Zha XF, Du H (2006) Knowledge-intensive collaborative design modeling and support: Part I: review, distributed models and framework. Comput Ind 57(1):39-55

Open Access This chapter is licensed under the terms of the Creative Commons Attribution 4.0 International License (http://creativecommons.org/licenses/by/4.0/), which permits use, sharing, adaptation, distribution and reproduction in any medium or format, as long as you give appropriate credit to the original author(s) and the source, provide a link to the Creative Commons license and indicate if changes were made.

The images or other third party material in this chapter are included in the chapter's Creative Commons license, unless indicated otherwise in a credit line to the material. If material is not included in the chapter's Creative Commons license and your intended use is not permitted by statutory regulation or exceeds the permitted use, you will need to obtain permission directly from the copyright holder.

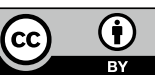

